\title{
A Note on the Tail Behavior of Randomly Weighted Sums with Convolution-Equivalently Distributed Random Variables
}

\author{
Yang Yang, ${ }^{1,2}$ Jun-feng Liu, ${ }^{1}$ and Yu-lin Zhang ${ }^{2}$ \\ ${ }^{1}$ School of Mathematics and Statistics, Nanjing Audit University, Nanjing 210029, China \\ ${ }^{2}$ School of Economics and Management, Southeast University, Nanjing 210096, China \\ Correspondence should be addressed to Yang Yang; yyangmath@gmail.com
}

Received 29 October 2013; Accepted 11 November 2013

Academic Editor: Litan Yan

Copyright (c) 2013 Yang Yang et al. This is an open access article distributed under the Creative Commons Attribution License, which permits unrestricted use, distribution, and reproduction in any medium, provided the original work is properly cited.

We investigate the tailed asymptotic behavior of the randomly weighted sums with increments with convolution-equivalent distributions. Our obtained result can be directly applied to a discrete-time insurance risk model with insurance and financial risks and derive the asymptotics for the finite-time probability of the above risk model.

\section{Introduction and Main Result}

Let $\left\{X_{n}, n \geq 1\right\}$ be a sequence of independent and identically distributed (i.i.d.) real-valued random variables with common distribution $F$, and let $\left\{Y_{n}, n \geq 1\right\}$ be another sequence of i.i.d. nonnegative r.v.s with common distribution $G$ and right endpoint $\widehat{x}_{G}=\sup \left\{x: \mathbb{P}\left(Y_{1} \leq x\right)<1\right\}$. Assume that $\left\{X_{n}, n \geq 1\right\}$ are independent of $\left\{Y_{n}, n \geq 1\right\}$. In this paper, we are interested in the randomly weighted sum

$$
S_{n}^{Y}=\sum_{i=1}^{n} X_{i} \prod_{j=1}^{i} Y_{j}, \quad n \geq 1 .
$$

This is because the study for the tail probability $\mathbb{P}\left(S_{n}^{Y}>x\right)$ can be directly applied to risk theory. Consider a discretetime insurance risk model. Within period $i, i \geq 1$, the net insurance loss is denoted by a real-valued (r.v.) $X_{i}$. The insurer makes both risk-free and risky investments, leading to an overall stochastic discounted factor $Y_{i}$ from time $i$ to time $i-1$. In the terminology of Norberg [1], the sequences $\left\{X_{n}, n \geq\right.$ $1\}$ and $\left\{Y_{n}, n \geq 1\right\}$ are called the insurance and financial risks, respectively. Then, the randomly weighted sum $S_{n}^{Y}$ in (1) represents the stochastic discounted value of aggregate net losses up to time $n, n \geq 1$. As usual, the probability of ruin by time $n$ can be defined by

$$
\Psi(x, n)=\mathbb{P}\left(\max _{1 \leq m \leq n} \sum_{i=1}^{m} X_{i} \prod_{j=1}^{i} Y_{j}>x\right), \quad n \geq 1,
$$

where $x \geq 0$ is interpreted as the initial capital reserve of an insurance company. Clearly, for each $n \geq 1$,

$$
\mathbb{P}\left(S_{n}^{Y}>x\right) \leq \Psi(x, n) \leq \mathbb{P}\left(\sum_{i=1}^{n} X_{i}^{+} \prod_{j=1}^{i} Y_{j}>x\right),
$$

where $X_{i}^{+}=X_{i} \mathbf{1}_{\left\{X_{i} \geq 0\right\}}$ denotes the positive part of $X_{i}, i \geq$ 1. If we can establish an asymptotic formula for $\mathbb{P}\left(S_{n}^{Y}>x\right)$ while doing so does not require $F(0-)>0$, then the same asymptotic formula should hold for the right-hand side of (3) as well. In this way the ruin probability $\Psi(x, n)$ has the same asymptotic behavior as that of the tail probability $\mathbb{P}\left(S_{n}^{Y}>x\right)$ as $x$ tends to infinity.

There has been a vast amount of literature studying the asymptotic behavior of the tail probability of the randomly weighted sum $S_{n}^{Y}$. Many works have considered the heavytailed case; that is, the distribution $F$ of $X$ belongs to some classes of heavy-tailed distributions, even under some 
dependence structures. For example, one can refer to Tang and Tsitsiashvili [2, 3], Wang and Tang [4], Zhang et al. [5], Shen et al. [6], Chen and Yuen [7], Gao and Wang [8], and Yi et al. [9] among others for some details in this direction, where the distribution $F$ is heavily heavy tailed; as for some lightly heavy-tailed distribution $F$, some related results were obtained by Tang and Tsitsiashvili $[3,10]$, Chen and Su [11], Hashorva et al. [12], Yang et al. [13], Yang and Hashorva [14], and Yang and Wang [15] among others. We pointed out that Tang and Tsitsiashvili [3] achieved some interesting results on the asymptotics for the tail probability $\mathbb{P}\left(S_{n}^{Y}>x\right)$ in some cases where $F$ belongs to the intersection between the subexponential distribution class and the rapidly varying distribution class.

In this paper, we aim to consider the light-tailed case, more exactly, to investigate the asymptotic behavior of the tail probability of the randomly weighted sums with increments with convolution-equivalent distributions.

Hereafter, all the limit relationships hold for $x$ tending to infinity. For two positive functions $a(x)$ and $b(x)$, we write $a(x) \sim b(x)$ if $\lim a(x) / b(x)=1$; write $a(x)=$ $o(b(x))$ if $\lim a(x) / b(x)=0$; and write $a(x)=O(b(x))$ if $\lim \sup a(x) / b(x)<\infty$.

Firstly we introduce some definitions on some classes of convolution-equivalent distributions. A distribution $V$ on $[0, \infty)$ belongs to the class of convolution-equivalent distributions, denoted by $\mathcal{S}(\gamma), \gamma \geq 0$, if for any $y \in \mathbb{R}$,

$$
\begin{gathered}
\lim \frac{\bar{V}(x-y)}{\bar{V}(x)}=e^{\gamma y}, \\
\lim \frac{\overline{V^{* 2}}(x)}{\bar{V}(x)}=2 \int_{0}^{\infty} e^{\gamma u} V(d u)<\infty,
\end{gathered}
$$

where $V^{* 2}$ denotes the convolution of $V$ with itself. More generally, a distribution $V$ on $\mathbb{R}$ belongs to the class $\delta(\gamma), \gamma \geq$ 0 , if and only if its right-hand distribution $V^{+}(x)=V(x) \mathbf{1}_{\{x \geq 0\}}$ belongs to this class; see Corollary 2.1 of Pakes [16]. The class $\mathcal{S}:=\mathcal{S}(0)$ is called the class of subexponential distributions. A distribution $V$ on $\mathbb{R}$ belongs to the class $\mathscr{L}(\gamma), \gamma \geq 0$ if only relation (4) holds. In the case $\gamma=0$, we say that $\mathscr{L}:=\mathscr{L}(0)$ is the class of long-tailed distributions. Similarly, a positive function $f(\cdot)$ is said to be long tailed if $\lim f(x-$ $y) / f(x)=1$ for any $y \in \mathbb{R}$. Clearly, if a distribution $V \in \mathscr{L}$, then its tail probability $\bar{V}(x)$ is long tailed. Closely related is the class $\mathscr{A}$, which was introduced by Konstantinides et al. [17]. A distribution $V$ on $\mathbb{R}$ belongs to the class $\mathscr{A}$ if $V$ is subexponential, and, for some $y>1$,

$$
\lim \sup \frac{\bar{V}(x y)}{\bar{V}(x)}<1 .
$$

Clearly, all distributions in the classes $\mathscr{A}, \mathcal{S}$, and $\mathscr{L}$ are heavy tailed. A distribution $V$ of r.v. $\xi$ is said to be heavy tailed if $\mathbb{E} e^{s \xi}=\infty$ for any $s>0$; otherwise it is said to be light tailed.

For each $n \geq 1$, denote the distribution of $X_{n} \prod_{j=1}^{n} Y_{j}$ by $H_{n}$, by convention, $H=H_{1}$. Now we state our main result as follows.
Theorem 1. If $F \in \mathcal{S}(\gamma)$ for some $\gamma>0, \widehat{x}_{G}=\infty$, and, for all $u>0$,

$$
\bar{G}(u x)=o(\bar{H}(x)),
$$

then, for each $n \geq 1$,

$$
\mathbb{P}\left(S_{n}^{Y}>x\right) \sim \overline{H_{n}}(x) .
$$

Remark 2. We remark that Tang [18] considered a similar result for $0<\widehat{x}_{G}<\infty$, whereas Theorem 1 deals with the case with $\widehat{x}_{G}=\infty$ for a complement.

Remark 3. In Theorem 1, relation (7) is a mild condition. According to Corollary 1.1 of Tang [19], relation (7) can be further implied by either
(a) $\bar{G}(v x)=o(\bar{G}(x))$ for some $v>1$ or
(b) $\bar{G}(v x)=o(\bar{F}(x))$ for some $v>0$.

\section{Proof of the Main Result}

We start this section by a series of lemmas. The first two lemmas are due to Lemma 3.2 and Theorem 2.1 of Tang [20].

Lemma 4. For two distributions $G$ and $H$ with $\bar{G}(x)>0$ and $\bar{H}(x)>0$ for all $x \geq 0$, relation (7) holds for each $u>0$, if and only if there is a nonnegative function $a(\cdot)$ such that

$$
a(x) \nearrow \infty, \quad \frac{a(x)}{x} \searrow 0, \quad \bar{G}(a(x))=o(\bar{H}(x)) .
$$

Lemma 5. Consider the product XY. The distribution $H$ of the product belongs to the class $\mathscr{A}$ if and only if $F \in \mathscr{A}$ and relation (7) holds for all $u>0$.

Tang [19] obtained an interesting result to show that a light-tailed random variable can be transferred into a heavytailed one through multiplier.

Lemma 6. Consider the product $X Y$ with $F \in \mathcal{S}(\gamma)$ for some $\gamma>0$ and $\widehat{x}_{G}=\infty$. If relation (7) holds for all $u>0$, then $H \in \mathcal{S}$.

The last lemma can be found in, for example, Theorem 3.14 of Foss et al. [21].

Lemma 7. Let a reference distribution $V$ on $\mathbb{R}$ belong to the class $\mathcal{S}$. Assume that distributions $V_{1}, \ldots, V_{n}$ on $\mathbb{R}$ satisfy that, for each $i=1, \ldots, n$, the function $\bar{V}+\bar{V}_{i}$ is long tailed and $\bar{V}_{i}(x)=O(\bar{V}(x))$. Then, it holds that

$$
\overline{V_{1} * \cdots * V_{n}}(x)=\overline{V_{1}}(x)+\cdots+\overline{V_{n}}(x)+o(\bar{V}(x)) .
$$

Proof of Theorem 1. Now we begin to prove the main result of Theorem 1.

For each $n \geq 1$, write

$$
T_{n}:=\sum_{i=1}^{n} X_{i} \prod_{j=i}^{n} Y_{j} \stackrel{d}{=} S_{n}^{Y},
$$


where $\stackrel{d}{=}$ stands for equality in distribution. Since $F \in \mathcal{S}(\gamma) \subset$ $\mathscr{L}(\gamma), \gamma>0$, its tail distribution $\bar{F}$ is rapidly varying in the sense that

$$
\lim \frac{\bar{F}(x y)}{\bar{F}(x)}=0, \quad \forall y>1 .
$$

By Lemma 6, we get that $H \in \mathcal{S}$. Further, by Lemma 4, there exists a nonnegative function $a(\cdot)$ such that (9) holds. Thus, by (9) and (12), for any $y>1$,

$$
\begin{aligned}
\lim \sup & \frac{\overline{H^{+}}(x y)}{\overline{H^{+}}(x)} \\
& =\lim \sup \frac{\left(\int_{0}^{a(x)}+\int_{a(x)}^{\infty}\right) \bar{F}(x y / u) G(\mathrm{~d} u)}{\overline{H^{+}}(x)} \\
& \leq \lim \sup \frac{\int_{0}^{a(x)} \bar{F}(x y / u) G(\mathrm{~d} u)}{\int_{0}^{a(x)} \bar{F}(x / u) G(\mathrm{~d} u)}+\lim \sup \frac{\bar{G}(a(x))}{\overline{H^{+}}(x)} \\
& \leq \lim \sup \frac{\sup }{0 \leq u \leq a(x)} \frac{\bar{F}(x y / u)}{\bar{F}(x / u)} \\
& =\lim \sup \frac{\bar{F}(x y)}{\bar{F}(x)}=0<1,
\end{aligned}
$$

which, together with $H \in \mathcal{S}$, implies that $H \in \mathscr{A}$.

We proceed to prove relation (8) by induction on $n$. Trivially, the distribution $H_{1}=H$ of $T_{1}$ or $S_{1}$ belongs to the class $\mathscr{A}$, and relation (8) holds for $n=1$. Assume that $H_{n} \in \mathscr{A}$ and (8) holds for $n$. We aim to prove that $H_{n+1} \in \mathscr{A}$ and (8) holds for $n+1$, which, by (11), is equivalent to

$$
\mathbb{P}\left(T_{n+1}>x\right) \sim \overline{H_{n+1}}(x) .
$$

First of all, according to Lemma 2.17 of Foss et al. [21] and $H_{n} \in \mathscr{A} \subset \mathscr{L}$, we have, that for any $s>0$,

$$
\lim \overline{H_{n}}(x) e^{s x}=\infty,
$$

which, together with $F \in \mathcal{S}(\gamma), \gamma>0$, implies that

$$
\bar{F}(x)=o\left(\overline{H_{n}}(x)\right) .
$$

By (16) and $H_{n} \in \mathscr{A} \subset \mathscr{L}$, we have that for any $y \in \mathbb{R}$,

$$
\begin{aligned}
\lim \frac{\overline{H_{n}}(x-y)+\bar{F}(x-y)}{\overline{H_{n}}(x)+\bar{F}(x)} \\
=\lim \left(\left(\frac{\overline{H_{n}}(x-y)}{\overline{H_{n}}(x)}\right)+\left(\frac{\bar{F}(x-y)}{\overline{H_{n}}(x-y)}\right)\right. \\
\left.\cdot\left(\frac{\overline{H_{n}}(x-y)}{\overline{H_{n}}(x)}\right)\right) \times\left(1+\left(\frac{\bar{F}(x)}{\overline{H_{n}}(x)}\right)\right)^{-1}
\end{aligned}
$$

$=1$, which shows that the function $\overline{H_{n}}(x)+\bar{F}(x)$ is long tailed. Since $T_{n}$ and $X_{n+1}$ are independent of each other, thus, by $H_{n} \in \mathscr{A} \subset \mathcal{S}$, we can apply Lemma 7 to derive from the induction assumption and (16) that

$$
\begin{aligned}
\mathbb{P}\left(T_{n}+X_{n+1}>x\right) & =\overline{H_{n}}(x)+\bar{F}(x)+o\left(\overline{H_{n}}(x)\right) \\
& =(1+o(1)) \overline{H_{n}}(x) .
\end{aligned}
$$

For the above-mentioned nonnegative function $a(\cdot)$, from (9) and (18), we obtain that

$$
\begin{aligned}
\mathbb{P}\left(T_{n+1}>x\right) \\
\quad=\mathbb{P}\left(\left(T_{n}+X_{n+1}\right) Y_{n+1}>x\right) \\
\quad=\left(\int_{0}^{a(x)}+\int_{a(x)}^{\infty}\right) \mathbb{P}\left(T_{n}+X_{n+1}>\frac{x}{u}\right) G(\mathrm{~d} u) \\
=(1+o(1)) \int_{0}^{a(x)} \overline{H_{n}}\left(\frac{x}{u}\right) G(\mathrm{~d} u)+o(\bar{H}(x)) \\
=(1+o(1)) \overline{H_{n+1}}(x),
\end{aligned}
$$

where the last step used the fact that $\bar{H}(x)=O\left(\overline{H_{n+1}}(x)\right)$, because, for any $x \geq 0$,

$$
\begin{aligned}
\overline{H_{n+1}}(x) & \geq \mathbb{P}\left(X_{n+1} \prod_{j=1}^{n+1} Y_{j}>x, Y_{1}>1, \ldots, Y_{n}>1\right) \\
& \geq \bar{H}(x)(\bar{G}(1))^{n},
\end{aligned}
$$

and $\bar{G}(1)>0$ by $\widehat{x}_{G}=\infty$. Relation (19) means that (14) holds. Finally, by (9) and (20), we have that

$$
\bar{G}(a(x))=o\left(\overline{H_{n+1}}(x)\right)
$$

from which and $H_{n} \in \mathscr{A}$, Lemma 5 gives that $H_{n+1} \in \mathscr{A}$.

This completes the proof of Theorem 1 .

\section{Conflict of Interests}

The authors declare that there is no conflict of interests regarding the publication of this paper.

\section{Acknowledgments}

The authors are most grateful to the two referees and the editor for their very thorough reading of the paper and valuable suggestions to improve the presentation of this paper. This research is supported by the National Natural Science Foundation of China (nos. 11001052 and 71171046), China Postdoctoral Science Foundation (no. 2012M520964), the Natural Science Foundation of Jiangsu Province of China (no. BK20131339), Qing Lan Project, and Project of Construction for Superior Subjects of Statistics \& Audit Science and Technology of Jiangsu Higher Education Institutions. 


\section{References}

[1] R. Norberg, "Ruin problems with assets and liabilities of diffusion type," Stochastic Processes and Their Applications, vol. 81, no. 2, pp. 255-269, 1999.

[2] Q. Tang and G. Tsitsiashvili, "Precise estimates for the ruin probability in finite horizon in a discrete-time model with heavy-tailed insurance and financial risks," Stochastic Processes and Their Applications, vol. 108, no. 2, pp. 299-325, 2003.

[3] Q. Tang and G. Tsitsiashvili, "Finite- and infinite-time ruin probabilities in the presence of stochastic returns on investments," Advances in Applied Probability, vol. 36, no. 4, pp. 12781299, 2004.

[4] D. Wang and Q. Tang, "Tail probabilities of randomly weighted sums of random variables with dominated variation," Stochastic Models, vol. 22, no. 2, pp. 253-272, 2006.

[5] Y. Zhang, X. Shen, and C. Weng, "Approximation of the tail probability of randomly weighted sums and applications," Stochastic Processes and Their Applications, vol. 119, no. 2, pp. 655-675, 2009.

[6] X.-m. Shen, Z.-y. Lin, and Y. Zhang, "Uniform estimate for maximum of randomly weighted sums with applications to ruin theory," Methodology and Computing in Applied Probability, vol. 11, no. 4, pp. 669-685, 2009.

[7] Y. Chen and K. C. Yuen, "Sums of pairwise quasi-asymptotically independent random variables with consistent variation," Stochastic Models, vol. 25, no. 1, pp. 76-89, 2009.

[8] Q. Gao and Y. Wang, "Randomly weighted sums with dominated varying-tailed increments and application to risk theory," Journal of the Korean Statistical Society, vol. 39, no. 3, pp. 305314, 2010.

[9] L. Yi, Y. Chen, and C. Su, "Approximation of the tail probability of randomly weighted sums of dependent random variables with dominated variation," Journal of Mathematical Analysis and Applications, vol. 376, no. 1, pp. 365-372, 2011.

[10] Q. Tang and G. Tsitsiashvili, "Randomly weighted sums of subexponential random variables with application to ruin theory," Extremes, vol. 6, no. 3, pp. 171-188, 2003.

[11] Y. Chen and C. Su, "Finite time ruin probability with heavytailed insurance and financial risks," Statistics \& Probability Letters, vol. 76, no. 16, pp. 1812-1820, 2006.

[12] E. Hashorva, A. G. Pakes, and Q. Tang, "Asymptotics of random contractions," Insurance, vol. 47, no. 3, pp. 405-414, 2010.

[13] Y. Yang, R. Leipus, and J. Šiaulys, “Tail probability of randomly weighted sums of subexponential random variables under a dependence structure," Statistics \& Probability Letters, vol. 82, no. 9, pp. 1727-1736, 2012.

[14] Y. Yang and E. Hashorva, "Extremes and products of multivariate AC-product risks," Insurance, vol. 52, no. 2, pp. 312-319, 2013.

[15] Y. Yang and Y. Wang, "Tail behavior of the product of two dependent random variables with applications to risk theory," Extremes, vol. 16, no. 1, pp. 55-74, 2013.

[16] A. G. Pakes, "Convolution equivalence and infinite divisibility," Journal of Applied Probability, vol. 41, no. 2, pp. 407-424, 2004.

[17] D. Konstantinides, Q. Tang, and G. Tsitsiashvili, "Estimates for the ruin probability in the classical risk model with constant interest force in the presence of heavy tails," Insurance, vol. 31, no. 3, pp. 447-460, 2002.

[18] Q. Tang, "On convolution equivalence with applications," Bernoulli, vol. 12, no. 3, pp. 535-549, 2006.
[19] Q. Tang, "From light tails to heavy tails through multiplier," Extremes, vol. 11, no. 4, pp. 379-391, 2008.

[20] Q. Tang, "The subexponentiality of products revisited," Extremes, vol. 9, no. 3-4, pp. 231-241, 2006.

[21] S. Foss, D. Korshunov, and S. Zachary, An Introduction to HeavyTailed and Subexponential Distributions, Springer, New York, NY, USA, 2011. 


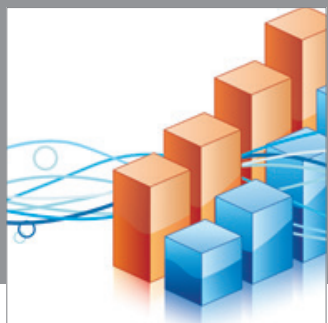

Advances in

Operations Research

mansans

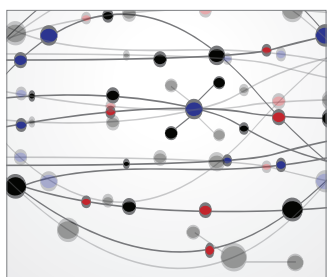

The Scientific World Journal
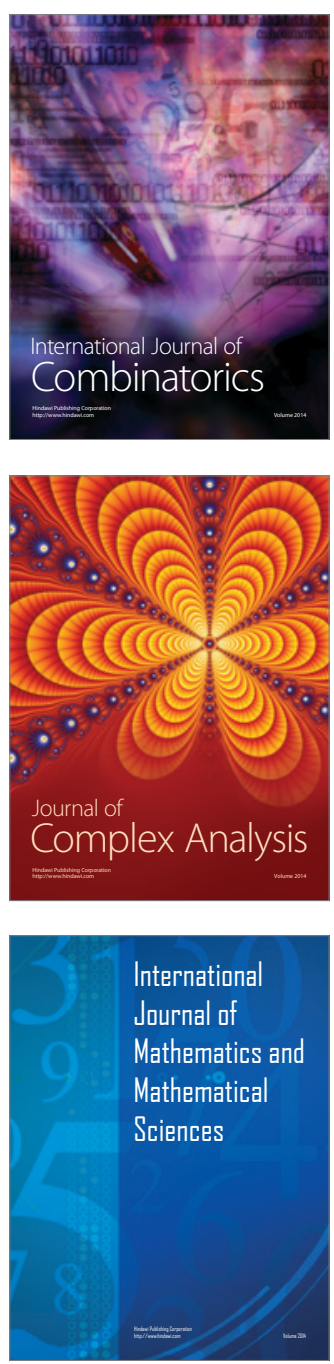
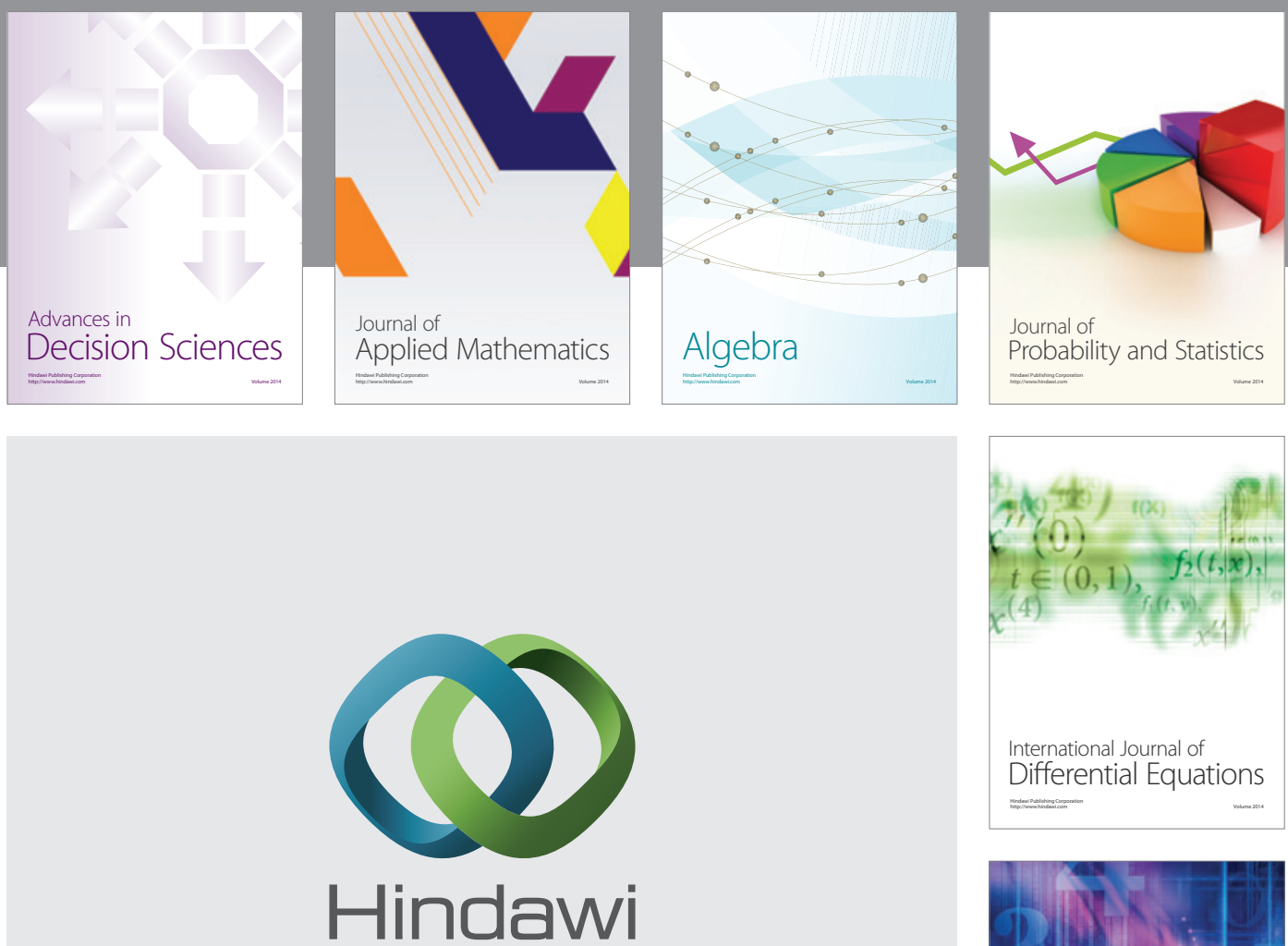

Submit your manuscripts at http://www.hindawi.com
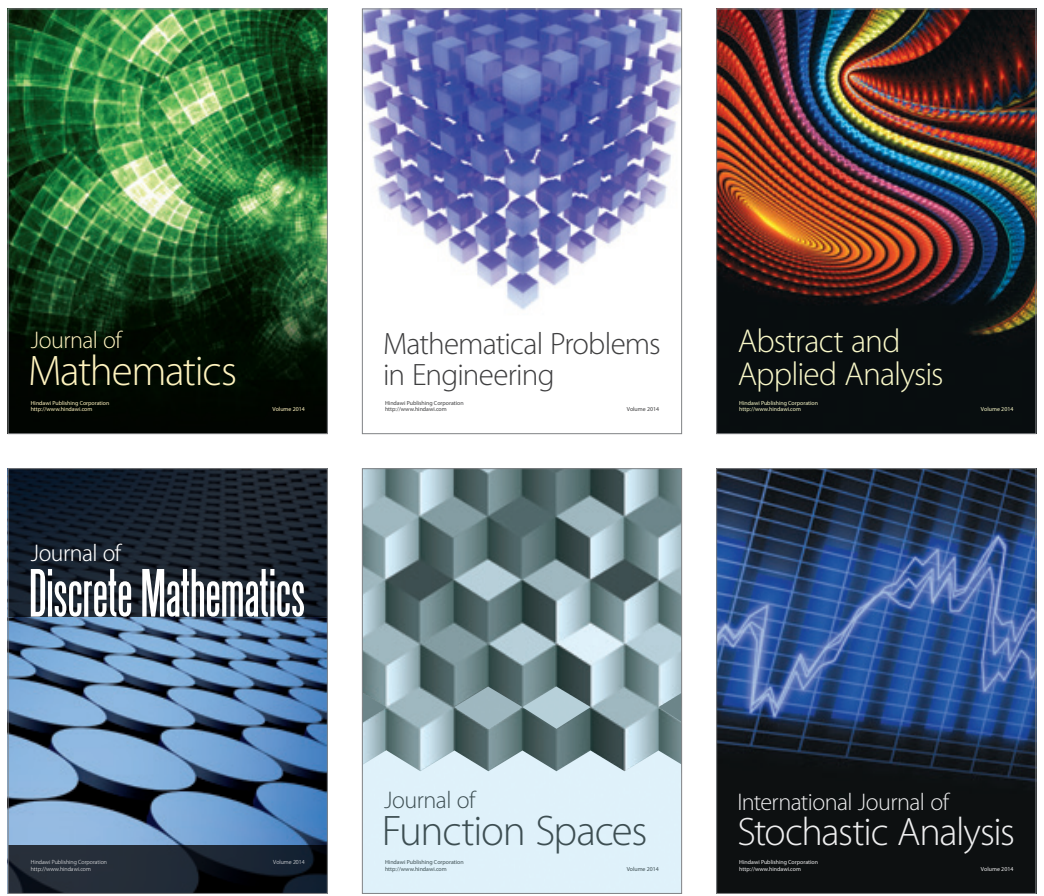

Journal of

Function Spaces

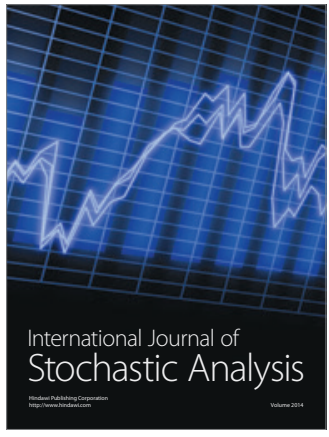

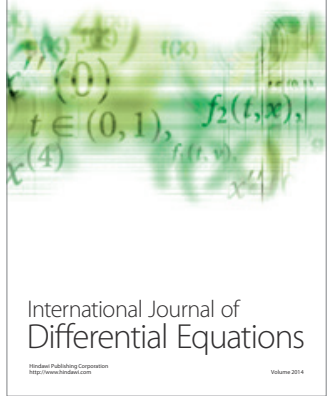
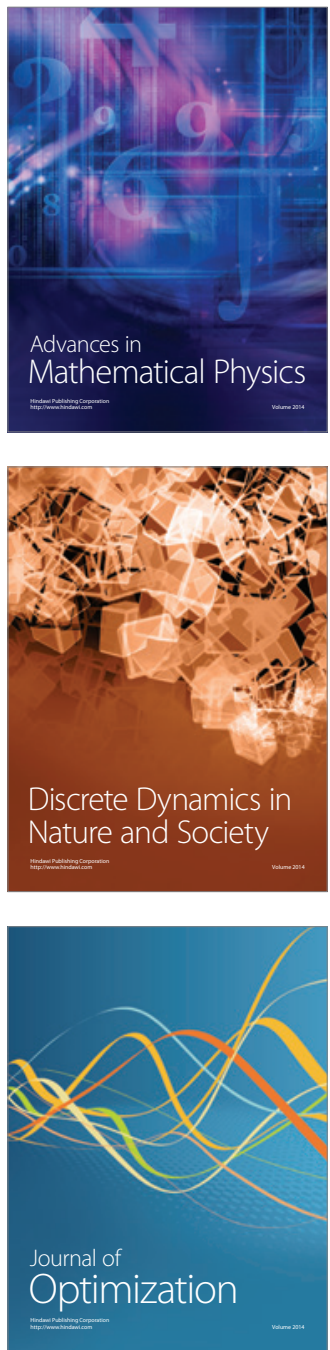